\title{
Serviço de atendimento farmacêutico ao idoso: perspectiva e propostas
}

\author{
Pharmaceutical care to the elderly: perspective and proposals
}

Recebido em: 13/10/2016

Aceito em: $\quad 13 / 03 / 2017$
Sandna Larissa Freitas dos SANTOS; Cinara Vidal PESSOA; Hérick Hebert da Silva ALVES; Romênio Nogueira BORGES;

Karla Bruna Nogueira Torres BARROS

Centro Universitário Católica de Quixadá- UNICATÓLICA. Rua Juvêncio Alves, 660,

Centro.CEP 63900-000. Quixadá, CE,Brasil.E-mail: sandy.lary@hotmail.com

\section{ABSTRACT}

The study aimed to investigate the prospect of a pharmacist and carry out educational activities for seniors in the House Remanso da Paz, Quixadá, CE, Brazil. It was an observational study, analytical, crossover, consisting of a quantitative and qualitative approach, carried out in June 2016 with 23 seniors (57\% women). The meetings occurred through informative lectures and educational activities, through a project of extension SAFI-Pharmaceutical care service to the elderly patient by Pharmacy students of the Catholic University Center of Quixadá. Among the topics discussed were: rational use of medications, use of homemade herbal preparations, diabetes, and hypertension, rheumatic problems, cardiovascular and respiratory diseases, and sedentary lifestyle/food. An interview was conducted with a corresponding social, economic profile of the elderly, and information about the prescribed medications and those used by the practice of self-medication. The elderly had 60 to age 81 years old, diagnosed with hypertension $(50 \%)$, gastritis $(19 \%)$, and Diabetes $(16 \%)$. The practice of self-medication was reported by $9(40 \%)$ elderly and was not mentioned adverse effect caused by the use of medicines. Among the total number of 10 medicines, $80 \%$ were NSAIDS with complaints of use between headaches and body. The pharmacist was seen as a source of guidance on the use of medicines in $13 \%$. With that, stresses the importance of the guidance of actions of the elderly quality of life and the importance of the role of the Pharmacist in the health of this population.

Keywords: pharmaceutical care; elderly; health education.

\section{RESUMO}

O estudo teve como objetivo averiguar a perspectiva do profissional farmacêutico em realizar ações educativas para idosos da casa de apoio Remanso da Paz, Quixadá, CE. Trata-se de um estudo observacional, analítico, transversal, consistindo em uma abordagem quanti-qualitativa, realizado no mês de junho de 2016 com 23 idosos (57\% mulheres). Os encontros aconteceram por meio de palestras informativas e educativas, através de um projeto de extensão SAFI Serviço de Atendimento Farmacêutico ao paciente idoso pelos estudantes de Farmácia do Centro Universitário Católica de Quixadá. Dentre os temas abordados estavam: uso racional de medicamentos, uso de preparações caseiras de plantas medicinais, diabetes e hipertensão, problemas reumáticos, doenças cardiovasculares e respiratórias, e sedentarismo/ alimentação. Foi realizada uma entrevista com um questionário correspondendo ao perfil sócio econômico dos idosos, e a informações sobre os medicamentos prescritos, bem como os utilizados pela prática da automedicação. Os idosos estavam na faixa etária entre 60 a 81 anos de idade, diagnosticados com hipertensão (50\%), gastrite (19\%), e diabetes $(16 \%)$. A prática da automedicação foi relatada por 9 (40\%) idosos e não foi mencionado efeito adverso causado pelo uso de medicamentos. Dentre o número total de 10 medicamentos $80 \%$ eram AINES com as queixas de uso entre dores de cabeça e no corpo. O farmacêutico foi visto como fonte de orientação sobre o uso de medicamentos em 13\%. Com isso, ficou ressaltada a importância da orientação de ações de qualidade de vida ao idoso e a importância da atuação do Farmacêutico na saúde desse estrato da população.

Palavras-chave: atenção farmacêutica; idoso; educação em saúde. 


\section{INTRODUÇÃO}

Idosos tendem utilizar de mais produtos farmacêuticos e apresentam particularidades farmacocinéticas e farmacodinâmicas que as tornam particularmente mais vulneráveis a efeitos adversos e interações medicamentosas. Em geral, com o aumento da idade cronológica, ocorre uma maior incidência de condições crônicas de saúde, o que predispõe os idosos a um maior consumo de medicamentos (1).

Fornecer Atenção Farmacêutica ao paciente idoso apresenta-se como uma maneira de amparar o prescritor e o paciente junto aos complexos fatores envolvidos com os problemas cardiovasculares. A intervenção farmacêutica, por meio de ações educativas e orientações sobre o regime terapêutico, traz benefícios à saúde do paciente e ao processo de promoção da saúde pública (2).

A amplitude da comunicação com os pacientes em Atenção Farmacêutica torna-se essencial para alcançar melhores resultados com as intervenções realizadas. Essa prática está baseada na interação considerável entre indivíduos, dentre eles, pacientes, familiares, profissionais de saúde e outros farmacêuticos, e tem sido vista como a maior indutora da satisfação do paciente em relação aos serviços de saúde (3).

Diante disso, o estudo teve como objetivo realizar ações educativas em saúde com idosos da casa de acolhida Remanso da Paz, Quixadá, CE por meio de um projeto de extensão SAFI - Serviço de Atendimento Farmacêutico ao paciente idoso, do curso de Farmácia do Centro Universitário Católica de Quixadá, além de verificar a perspectiva da atuação do profissional farmacêutico no cuidado da saúde do idoso.

\section{MATERIAL E MÉTODOS}

Trata-se de um estudo do tipo observacional, descritivo, transversal, consistindo em uma abordagem quanti-qualitativa. Foi realizado no mês de junho de 2016 numa casa de acolhida de idosos Remanso da Paz, Quixadá-CE, assistida por profissionais médicos, fisioterapeuta, nutricionista, enfermeiro, e de serviços gerais, contando com doações para manter suas ações com atividades ocupacionais e educativas, sendo mediadas por voluntários.

Os dados de interesse foram obtidos por meio de uma entrevista, que utilizou como instrumento um questionário, para traçar o perfil socioeconômico e averiguar a opinião da atuação do profissional farmacêutico no âmbito da saúde do idoso. O estudo contemplou 23 idosos, que participaram efetivamente dos encontros assistindo palestras informativas e educativas, utilizando materiais de demonstração e vídeos explicativos, realizadas por meio do projeto acadêmico do curso de Farmácia, denominado de Serviço de Atendimento Farmacêutico ao Idoso, do Centro Universitário Católica de Quixadá. Por fim, foi disponibilizado material educativo sobre qualidade de vida ao idoso e a importância da atuação do Farmacêutico na saúde desta população.

Os dados relativos às questões fechadas foram organizados em planilha no Microsoft Excel. As respostas das questões abertas foram agrupadas em categorias de acordo com as respectivas similaridades. Os resultados foram analisados em frequências simples e absolutas. As análises estatísticas foram realizadas pelo Teste Exato de Fisher, com significância para $\mathrm{p}<0,05$.

O idoso autorizou sua participação na pesquisa com o Termo de Consentimento Livre e Esclarecido, e foi aprovado pelo Comitê de Ética do Centro Universitário Católica de Quixadá pelo parecer 1.478.724, por meio da Plataforma Brasil, de acordo com a Resolução 466/12 do Conselho Nacional de Saúde, que regulamenta as diretrizes e normas da pesquisa em seres humanos (4), seguindo as determinações desta, que são especificidades das pesquisas com seres humanos.

\section{RESULTADOS E DISCUSSÃO}

Aidentificação dos idosos participantes está descrita na tabela 1. No total $13(57 \%)$ eram mulheres e $10(43 \%)$ homens, com faixa etária entre 60 a 81 anos de idade.

A prática da automedicação foi relatada por 9 $(40 \%)$ idosos e nenhum mencionou efeito negativo causado pelo medicamentos. Dentre o número total de 10 substâncias citadas, estavam 3 (30\%) paracetamol, 3 (30\%) dipirona, $1(10 \%)$ omeprazol, $1(10 \%)$ bromoprida, 1 (10\%) ibuprofeno, 1 (10\%) ácido acetilsalicílico. Sobre a classificação terapêutica, $80 \%$ referiam-se a anti-inflamatórios não-esteroidais (AINES), antieméticos $(10 \%)$ e antiulcerogênico (10\%).

O uso mais recorrente de medicamentos pela automedicação foi referido a paracetamol, dipirona e ibuprofeno, para dores de cabeça e no corpo; bromoprida, para náuseas e vômitos; e omeprazole, para azia (Tabela 2).

Todos os idosos afirmaram não ingerir bebidas alcoólicas e não praticar exercícios físicos, e apenas 3 (13\%) idosos relataram fumar. Quando indagados sobre a fonte de orientação sobre o uso de medicamentos 20 
(87\%) afirmaram consultar os médicos e 3 (13\%) o farmacêutico. $100 \%$ deles afirmaram não seguir uma alimentação saudável.

Tabela 1. Características sócio econômicas dos idosos atendidos na casa de repouso Remanso da Paz, Quixadá, CE (junho 2016).

\begin{tabular}{|c|c|c|}
\hline \multicolumn{3}{|c|}{ Caracteristicas sócio econômicas } \\
\hline Idade & Frequência & $\%$ \\
\hline 60 a 71 & 20 & $87 \%$ \\
\hline 72 a 81 & 3 & $13 \%$ \\
\hline \multicolumn{3}{|c|}{ Estado civil } \\
\hline Casados & 12 & $52 \%$ \\
\hline Viúvos & 7 & $30,5 \%$ \\
\hline Separados & 4 & 17,5 \\
\hline \multicolumn{3}{|c|}{ Escolaridade } \\
\hline $1^{\circ}$ grau completo & 8 & $35 \%$ \\
\hline $2^{\circ}$ grau completo & 3 & $13 \%$ \\
\hline Não alfabetizado & 12 & $52 \%$ \\
\hline \multicolumn{3}{|c|}{ Renda Familiar } \\
\hline 1 salário & 15 & $65 \%$ \\
\hline 2 salários & 8 & $35 \%$ \\
\hline \multicolumn{3}{|c|}{ Situação Habitacional } \\
\hline Alugada & 8 & $35 \%$ \\
\hline Própria & 15 & $65 \%$ \\
\hline
\end{tabular}

Tabela 2. Patologias descritas pelos idosos atendidos na casa de repouso Remanso da Paz, Quixadá, CE, (junho 2016)

\begin{tabular}{|c|c|c|}
\hline Patologia & Frequência & $\%$ \\
\hline Hipertensão & 19 & $50 \%$ \\
\hline Gastrite & 7 & $19 \%$ \\
\hline Diabetes & 6 & $16 \%$ \\
\hline Depressão & 3 & $7,5 \%$ \\
\hline Asma & 1 & $2,5 \%$ \\
\hline Artrose & 1 & $2,5 \%$ \\
\hline Acidente Vascular Encefálico & 1 & $2,5 \%$ \\
\hline
\end{tabular}

Quando indagados sobre a fonte de orientação sobre o uso de medicamentos, 20 (87\%) afirmaram consultar os médicos e 3 (13\%), o farmacêutico. Todos afirmaram não seguir uma alimentação saudável.
O uso de pelo menos um medicamento foi relatado por todos os entrevistados. De acordo com a Tabela 3, foram citados 46 princípios ativos prescritos aos idosos. De acordo com a classificação terapêutica dos medicamentos foram prescritos anti-hipertensivos (52\%), antiulcerogênicos (13,5\%), antidiabéticos (11,5\%), AINES $(10,5 \%)$, antilipidêmicos $(4,5 \%)$, antiasmáticos $(2 \%)$, antieméticos $(2 \%)$, anticonvulsivantes $(2 \%)$, e antidepressivos $(2 \%)$.

Em media, foram observados 2 medicamentos por idoso o que, levando em consideração o estrato da população estudada, indica um baixo índice de polimedicação e a ocorrência de efeitos adversos aos medicamentos não foi relatada pelos participantes.

Tabela 3. Medicamentos prescritos para idosos atendidos na casa de repouso Remanso da Paz, Quixadá, CE, (junho 2016).

\begin{tabular}{|c|c|c|}
\hline Medicamentos & Frequência & $\%$ \\
\hline Hidroclorotiazida & 8 & $17,5 \%$ \\
\hline Losartana & 7 & $15,5 \%$ \\
\hline Captropil & 5 & $11 \%$ \\
\hline Metformina & 4 & $8 \%$ \\
\hline Omeprazol & 3 & $7 \%$ \\
\hline Pantoprazol & 3 & $7 \%$ \\
\hline Metildopa & 2 & $5,5 \%$ \\
\hline Ácido acetilsalicílico & 2 & $5,5 \%$ \\
\hline Alodipino & 1 & $2 \%$ \\
\hline Ramipril & 1 & $2 \%$ \\
\hline Glicazida & 1 & $2 \%$ \\
\hline Salbutamol & 1 & $2 \%$ \\
\hline Domperidona & 1 & $2 \%$ \\
\hline Sinvastatina & 1 & $2 \%$ \\
\hline Aspirina & 1 & $2 \%$ \\
\hline Rivotril & 1 & $2 \%$ \\
\hline Rivastatina & 1 & $2 \%$ \\
\hline Dipirona & 1 & $2 \%$ \\
\hline Fluoxetina & 1 & $2 \%$ \\
\hline Meloxican & 1 & $2 \%$ \\
\hline
\end{tabular}

Todos os participantes afirmaram aderir ao tratamento fazendo uso de forma correta. Conforme a análise realizada utilizando os programas Drug Interaction Facts on Disc ${ }^{\circledR}(5)$ e (PR) Vade-Mécum ${ }^{\circledR}(6)$, não foram detectadas interações entre os medicamentos prescritos para idosos que afirmavam tomar mais de um medicamento.

O Quadro 1 mostra as respostas dos idosos quando questionados sobre a importância de ações educativas e do profissional farmacêutico na saúde do idoso. 
Quadro 1. Opinião sobre as ações educativas em saúde e a importância do farmacêutico na saúde dos idosos atendidos na casa de repouso Remanso da Paz, Quixadá, CE, (junho 2016)

\begin{tabular}{|c|c|}
\hline Perguntas & Respostas \\
\hline \multirow{4}{*}{$\begin{array}{l}0 \text { Sr(a) acredita ser importante } \\
\text { ações educativas em saúde para a } \\
\text { população idosa? }\end{array}$} & $\begin{array}{l}\text { Sim, eu participei deste o primeiro dia que vocês "vinheram", e aprendi muita coisa que não sabia... } \\
\text { (F.C.v.S/68 anos) }\end{array}$ \\
\hline & $\begin{array}{l}\text { Demais,.. Eu aprendi sobre as plantas, as comidas..Sem contar sobre as doenças, muita coisa a gente não } \\
\text { sabia... (M.V.S/74 anos) }\end{array}$ \\
\hline & $\begin{array}{l}\text { Eu acredito, com essas palestras a gente fica sabendo dessas novas doenças que aparecem, e do que } \\
\text { comer pra não se sentir mal... (F.L.F/72 anos) }\end{array}$ \\
\hline & $\begin{array}{l}\text { Só ensinando assim, pra gente aprender...principalmente a gente que é hipertensos e diabéticos, que toma } \\
\text { vários remédios, "é" muito importantes esses encontros... (F.E.C.F/83 anos) }\end{array}$ \\
\hline \multirow{4}{*}{$\begin{array}{l}\text { Em que o } \operatorname{Sr}(a) \text { acredita que o } \\
\text { farmacêutico pode ajudar na saúde } \\
\text { do idosos? }\end{array}$} & Em ajudar a gente a tomar os remédios corretos, na hora correta e com aguá... (F.I.L/79 anos) \\
\hline & $\begin{array}{l}\text { Eu aprendi a tomar o remédio com água e não com suco, e agora sempre faço isso... E foi um farmacêuti- } \\
\text { co que me falou... }\end{array}$ \\
\hline & $\begin{array}{l}\text { "Eles deixam o medicamento mais fácil", eu não sabi a hora de tomar...e ainda falam sobre o que comer } \\
\text { para não ter efeito.." "né"... (F.E.F.C/79 anos) }\end{array}$ \\
\hline & Explicando direito como tomar os remédios e agora eu não esqueço mais... (F.L.T/79 anos) \\
\hline
\end{tabular}

A predominância do sexo feminino $(68,0 \%)$ na instituição pesquisada demonstra o fenômeno de feminização da velhice, decorrente da maior expectativa de vida da mulher no Brasil (7). Muitos programas de promoção da saúde apresentam fortes marcas da feminização, as quais podem provocar nos homens uma sensação de não pertencimento e os tornarem menos familiarizados com o espaço e a lógica de organização destes serviços (8).

Outro aspecto a ser considerado refere-se à faixa etária (60 a 71 anos) mais prevalente, pois à medida que a idade avança, aumentam os riscos de adoecer e apresentar maior grau de dependência e, consequentemente, maior utilização de medicamentos. Em um estudo realizado em Belo Horizonte, MG, foi observado um maior número de idosos institucionalizados na faixa dos 80 anos ou mais (50,0\%), seguido por aqueles entre 70 a 79 $\operatorname{anos}(34,1 \%)(9)$.

Nos últimos anos, a perspectiva de vida tem crescido e com isso a quantidade de idosos, aumentando o índice populacional e a procura por medicamentos para uma melhor qualidade de vida. Isso se deve às ações de saúde pública como vacinação, saneamento, programas de prevenção, promoção da saúde do idoso, além dos avanços técnicos científicos das ciências da saúde. Com a senescência, a vulnerabilidade do idoso às doenças crônicas tendem a ter um aumento significativo, devido às modificações das funções fisiológicas do organismo. Isso acarreta em uso simultâneo de vários medicamentos (polifármacos) na tentativa de diminuir tais alterações (10).

Lima e Duarte (2013) avaliaram 62 idosos, sendo $98,3 \%$ do sexo feminino e observaram que as duas doenças crônicas associadas foram hipertensão arterial sistêmica (HAS, 44\%), HAS e Diabetes Mellitus (13\%) e 39\% não referia-se a patologias (11), semelhantes aos dados do presente estudo, no qual $50 \%$ apresentaram hipertensão.

Somados às comorbidades crônicas, são vários os fatores que levam à polifarmácia, entre os quais se destacam: acesso facilitado a medicamentos, características do médico e do paciente, reduzida frequência de uso de meios não-farmacológicos para os problemas de saúde. É essencial a minimização da polifarmácia, pois quanto maior o número de medicamentos utilizados maior é a chance de interações medicamentosas, salientando-se que nas fases de testes de ensaios clínicos feitos com fármacos a serem comercializados, a população idosa é muitas vezes excluída, de modo que as informações sobre a eficácia e a segurança do uso nesse grupo acabam tornando-se escassas (12).

A prática da polifarmácia em muitos casos é justificada pelo quadro de doenças apresentado pelo indivíduo, e pode fornecer benefício no controle de muitas condições crônicas. Ela tem sido definida de diferentes formas. A definição qualitativa é a prescrição, administração ou uso de outros medicamentos, relacionados com os que estão clinicamente indicados ao paciente. A definição quantitativa da polifarmácia apresenta várias classificações, partindo desde o uso de dois ou mais medicamentos, até o uso de seis ou mais. Há ainda a classificação para polifarmácia subdividindo-a como polifarmácia menor - uso de 2 a 4 medicamentos; e polifarmácia maior - uso de 5 ou mais medicamentos. Ou ainda, polifarmácia baixa - 2 a 3 medicamentos; moderada 
- 4 a 5 medicamentos; e alta - mais que 5. Ainda, em Grupo 1-3, 4-5, 6-8, e mais que 9 medicamentos (13).

Para entender e prevenir a polifarmácia, além de tratar suas complicações, o profissional de saúde que atende o público geriátrico deve ter conhecimento que abarque desde as alterações orgânicas próprias do envelhecimento que irão influenciar no metabolismo dos fármacos, até a farmacologia das medicações prescritas, suas possíveis interações medicamentosas e efeitos adversos, as dificuldades encontradas pelo idoso em orientar-se nas posologias e a dificuldade da adesão terapêutica, e ainda, a realidade socioeconômica individual desses pacientes (14). No presente estudo foram observados 2 medicamentos por idoso o que indica um baixo índice de polimedicação, levando em consideração que a população idosa apresenta maiores condições patológicas e como consequência elevado número de medicamentos. Contudo, o estudo retrata a minimização de efeitos adversos e interações medicamentosas.

Em comparação aos dados do presente estudo, a pesquisa de Galato e cols (2010) encontrou dados diferentes na avaliação da polimedicação, o qual observou que $20(19,3 \%)$ entrevistados não eram polimedicados (utilizavam até um medicamento), 54 (51,9\%) classificaram-se como polimedicação menor (uso de dois a quatro medicamentos) e $30(28,8 \%)$ classificaram-se como polimedicação maior (cinco medicamentos ou mais) (15).

Na prática clínica da polimedicação, os farmacêuticos estão aptos para orientar a respeito dos medicamentos prescritos e dispensados aos idosos, pois estão em contato frequente com os pacientes, podendo iniciar discussões sobre os problemas de saúde, informar sobre a natureza da doença crônica e identificar as razões do tratamento (7). A participação dos farmacêuticos no controle da hipertensão arterial, tida como a patologia mais prevalente na população estudada $(50 \%)$, consiste na seleção, gerenciamento do estoque, do armazenamento correto e na dispensação dos medicamentos, mas, principalmente, na promoção da Atenção Farmacêutica ao paciente (15).

A ausência da ingestão de bebidas alcoólicas reflete positivamente no contexto de saúde e no desenvolvimento do tratamento medicamentoso; porém, a falta de prática de exercícios físicos, de uma alimentação saudável e o ato de fumar desfavorecem a melhora em distúrbios cardíacos. A fonte de orientação sobre o uso de medicamentos pelo médico (87\%) é evidenciada pela maior busca pelos idosos por esses profissionais; o farmacêutico (13\%) é solicitado apenas na compra do medicamento.
Sobre os medicamentos mais utilizados pelos idosos estão hidroclorotiazida (17,5\%), losartana (15,5\%) e captropil (11\%) correlacionando com a hipertensão como a patologia mais prevalente. Dados semelhantes foram encontrados por Oliveira e Novaes (2012), que observaram os anti-hipertensivos como a classe dos mais prevalentes, sendo captopril (54,6\%), seguido pelos diuréticos tiazídicos (indapamida e hidroclorotiazida) $(39,6 \%) .(17)$

Todos os participantes afirmaram aderir ao tratamento de forma correta, associado à ausência de interações medicamentosas refletindo adequadamente nos resultados clínicos obtidos. No estudo de Silva e cols (2010), resultados diferentes foram encontrados: $73,4 \%$ das mulheres e $70,6 \%$ dos homens relataram o uso correto dos medicamentos (16).

De acordo com Secoli (2010), muitos medicamentos comumente usados por idosos, por exemplo, AINE, beta-bloqueadores, inibidores da enzima conversora de angiotensina (IECA), diuréticos, digoxina, antilipidêmicos, e depressores do sistema nervoso central, são potencialmente interativos (18). Há, ainda, os indutores (fenitoina, carbamazepina) e inibidores enzimáticos, por exemplo, cimetidine e omeprazol que, freqüentemente, encontram-se envolvidos nas interações medicamentosas, que ameaçam a saúde do idoso.

Na prática clínica, Atenção Farmacêutica é o seguimento farmacoterapêutico documentado do paciente, visando a terapia efetiva, por meio da prevenção, detecção e resolução dos problemas relacionados a medicamentos (PRM), além da melhoria da qualidade de vida. Os farmacêuticos atuam como último elo entre a prescrição e a administração, identificando, na dispensação, os pacientes de alto risco, enfatizando a importância da monitorização da farmacoterapia e controle da pressão arterial, evitando futuras complicações (19).

A educação em saúde é aquela que se baseia no diálogo e na troca de saberes, constituindo um verdadeiro intercâmbio entre o saber científico e o popular, de modo que cada um tem muito a ensinar e muito a aprender. A comunicação na atenção à saúde é algo que se constrói em uma ação intencional, dirigida e orientada para um interesse concreto. Os processos comunicativos baseiam-se em escutar o outro para compreender quais as suas crenças, sua situação e suas possibilidades e assim atuar conjuntamente (20).

No âmbito educativo, o estudo propôs, como estratégia, a abordagem dos variados temas relacionados à Geriatria, dentre eles: uso racional de medicamen- 
tos, uso de preparações caseiras de plantas medicinais, diabetes e hipertensão, problemas reumáticos, doenças cardiovasculares, sedentarismo/ alimentação, e doenças respiratórias. Ainda foi realizada verificação de pressão arterial dos participantes no decorrer dos encontros, para controle e avaliação das terapias.

O Quadro 1 mostra a intervenção favorável das ações educativas, além da atuação do profissional farmacêutico na conduta de estratégias de melhoria no estado clinico dos mesmos.

A educação, portanto, é um dos meios para vencer os desafios impostos aos idosos pela idade e pela sociedade, propiciando-lhes o aprendizado. Como exemplo, programas de educação em osteoporose aumentam o nível de conhecimento, atenção e cuidados com a saúde óssea, mostrando a necessidade de contínuo esforço em programas dessa natureza. O idoso deve obter informações sobre os aspectos da sua doença para conseguir refletir sobre sua saúde (21).

Assim, para fortalecer essa ação educativa, uma tecnologia educacional no formato impresso, tipo folder ou cartilha, desponta como dispositivo para mediar o modo de cuidar, por meio de estratégias para potencializar as capacidades do outro. Nesta perspectiva ampliada, a educação em saúde também capacita o farmacêutico a intervir de forma construtiva-reflexiva, singular/plural, dinâmica/flexível, num complexo histórico cultural de relações humanas entre sujeitos, num sistema cíclico de relações, em que um aprende com o outro; este aprender converge para a transformação de ambos, de quem os rodeia e do meio no qual estão inseridos (23). O desenvolvimento de ações educativas com idosos é uma maneira de ampliar o debate acerca dessas questões, bem como de favorecer a formação de profissionais com competência para cuidar nesse cenário (23).

Nesse contexto, nas falas dos entrevistados é destacada a importância de prestar ações educativas e orientações sobre o regime terapêutico como aspecto benéfico à saúde do paciente idoso e ao processo de promoção da saúde, apresentando estratégias facilitadoras para implantar o serviço de Atenção Farmacêutica ao idoso e que esses recursos sejam um referencial aos estabelecimentos de saúde.

\section{CONCLUSÃO}

A Atenção Farmacêutica está baseada no vinculo considerável de indivíduos, dentre eles, pacientes, familiares e profissionais de saúde, principalmente os farmacêuticos. Esta interação social tem sido vista como a maior indutora da satisfação do paciente em relação aos serviços de saúde e ao desenvolvimento do eficaz de um tratamento medicamentoso. De acordo com as falas apresentadas, foi observado que o projeto SAFI auxiliou na adesão medicamentosa aos idosos, possibilitando a transmissão de informações sobre as patologias e o uso racional de medicamento. Somada a isso, a contribuição à formação acadêmica dos estudantes de Farmácia, que puderam expor seu conhecimento na prática diária, prestando a Atenção Farmacêutica aos idosos, desenvolvendo uma dispensação medicamentosa eficaz.

Neste contexto, a importância da realização de atividades de educação em saúde à população idosa, está no ato de cuidar do ser idoso e de seu cuidador. As ações não dever ser focadas na patologia, mas priorizar a promoção, manutenção e recuperação da saúde. Com isso, ao valorizar as trocas interpessoais permeadas pelo diálogo, ressaltando a importância do conhecimento popular, as lacunas existentes nas práticas educativas de saúde tradicionais podem ser superadas, favorecendo um serviço farmacêutico qualificado.

\section{AGRADECIMENTOS}

Agradecemos o apoio do Remanso da Paz, Quixadá, CE, Brasil, aos voluntários da instituição e aos idosos que aceitaram participar, aos estudantes de Farmácia do Centro Universitário Católica de Quixadá, CE. 


\section{REFERÊNCIAS}

1. Aguiar PM, Lyra JDP, Silva DT, Marques TC. Avaliação da farmacoterapia de idosos residentes em instituições asilares no nordeste do Brasil. Lat. Am J Pharm. 2008; 27(3):454-459.

2. Lyra Júnior DP. Comunicação paciente/ farmacêutico: um instrumento libertário e essencial no trabalho do profissional e na promoção da saúde. Pharmacia Bras. 2005; 1(6): 10 .

3. Meneses ALL, Sá MLB. Atenção farmacêutica ao idoso: fundamentos e propostas. Geriatria \& Gerontol. 2010; 4(3):154-161.

4. BRASIL. Conselho Nacional de Saúde, Resolução n466 de 12 de dezembro de 2012.

5. Drug Interaction Facts on Disc [computer program]. Versão1.0.Medifor Inc;1999.

6. P. R. Vade-Mécum [programa para computador] Brasil. 2005-2006. Disponível em: http://p-r-vade-m-cumbrasil-2005-2006.software.informer.com/1.0/

7. BRASIL. Instituto Brasileiro de Geografia e Estatística. Indicadores Sociodemográficos e de Saúde no Brasil. Rio de Janeiro: Instituto Brasileiro de Geografia e Estatística; 2009. 152.

8. Couto MT, Pinheiro TF, Valença O, Machin R, Silva GSN, Gomes R, Schraiber LB, Figueiredo WS. O homem na atenção primária à saúde: discutindo (in)visibilidade a partir da perspectiva de gênero. Interface Comun Saúde Educ. 2010; 14(33):257-270

9. Ferreira RC, Magalhães CS, Rocha ES, Schwambach CW, Moreira AN. Saúde bucal de idosos residentes em instituições de longa permanência de Belo Horizonte, Minas Gerais, Brasil. Cad Saúde Pública. 2009; 25(11):23752385. DOI: 10.1590/S0102-311X2009001100008.

10. Almeida RO, Paiva CEQ, Ferreira SCH, Paiva YCS, Prado RMS. A importância da atenção farmacêutica para os pacientes geriátricos, Rev Expressão Católica, 2014; 6(3): 44-56.

11. Lima PV, Duarte SFP. Prevalência de obesidade em idosos e sua relação com Hipertensão e Diabetes. InterScientia, João Pessoa, 2013; 1(3):80-92.

12. Costa RM, Lima VAB, Paiva IG, Sousa PTP, Lima LG. Uso de medicamentos por idosos: algumas considerações Geriatria \& Gerontol. 2008; 3(2):126-131.
13. Lucchetti G, Granero AL, Pires SL, Gorzoni ML. Fatores associados à polifarmácia em idosos institucionalizados. Rev Bras Geriatr Gerontol. 2010; 13(1):51-58.

14. Silva DF, Schmidt OF, Silva S. Polifarmácia em geriatria. Rev AMRIGS, Porto Alegre, 2012; 56 (2): 164-174.

15. Galato D, Silva ES, Tiburcio LS. Estudo de utilização de medicamentos em idosos residentes em uma cidade do sul de Santa Catarina (Brasil): um olhar sobre a polimedicação. Ciênc Saúde Col. 2010; 15(6):2899-2905. DOI: 10.1590/S1413-81232010000600027.

16. Silva CSO, Pereira MI, Yoshitome AY, Neto JFR, Barbosa DA. Avaliação do uso de medicamentos pela população idosa em Montes Claros, Minas Gerais, Brasil. Esc Anna Nery (impr.). 2010; 14 (4):811-818.

17. Oliveira MP, Novaes MRCG. Uso de medicamentos por idosos de instituições de longa permanência, Brasília-DF, Brasil. Rev. bras. enferm. 2012; 65(5):737-744. DOI: 10.1590/S0034-71672012000500004.

18. Secoli SR. Polypharmacy: interaction and adverse reactions in the use of drugs by elderly people. Rev. bras. Enferm. 2010; 63(1):136-140. DOI: 10.1590/S003471672010000100023.

19. Forsetlund L, Eike MC, Gjerberg E, Gunn EV. Effect of interventions to reduce potentially inappropriate use of drugs in nursing homes: a systematic review of randomised controlled trials, BMC Geriatrics. 2011. DOI: 10.1186/1471-2318-11-16.

20. Marchon RM, Cordeiro RC, Nakano MM. Capacidade Funcional: estudo prospectivo em idosos residentes em uma instituição de longa permanência. Rev Bras Geriatr Gerontol. 2010; 13(2):203-214. DOI: 10.1590/S180998232010000200005.

21. Chrispino ML, Andrade M. A educação em saúde aos idosos atendidos pelo PSF. Informe-se em promoção da saúde, 2010; 6(1): 07-09.

22. Rodrigues D, Santos VE. Health Education in Family Health Strategy: a review of scientific publications in Brazil. J. Health Sci. Inst., 2010; 28(4). DOI: 10.5205/ reuol.8463-73861-2-SM.0910sup201525.

23. Brum DJT, Soccol KLS, Terra MG. Educação em saúde com idosos em grupos da "terceira idade". Anais Jornada Nacional de Educação, Santa Maria, RS, Educação : território de saberes. 2012. 